\title{
Condition monitoring and deterioration analysis of metal oxide varistor
}

\author{
Guoming Wang*, Woo-Hyun Kim*, Jong-Hyuk Lee ${ }^{* *}$, Gyung-Suk Kil ${ }^{*}$
}

\begin{abstract}
With the growing demand for application of metal-oxide varistor (MOV) in low-voltage electronic circuits for overvoltage protection, it is necessary to ensure its performance to avoid the short-circuit and the line-to-ground fault during operation. In this paper, a precise leakage current analyzer was developed to detect the total leakage current and third harmonic component of MOV for its condition monitoring. The voltage- and temperature-dependent measuring uncertainties were compensated using the multipliers. In addition, the deterioration characteristics of the MOV and the newly developed thermally protected metal-oxide varistor (TMOV) were investigated in the accelerated aging test. From the experimental results, the MOV deteriorated much faster under the lightning current impulse synchronized with power-frequency voltage. The thermally activated fuse of TMOV exploded under two types of impulses, which indicated that the TMOV is much more vulnerable and that it is difficult to diagnose the condition of energized TMOV in advance.
\end{abstract}

K e y w o r d s: metal-oxide varistor, thermal protected metal-oxide arrester, condition monitoring, deterioration analysis, accelerated aging test

\section{Introduction}

The metal-oxide varistor (MOV), which has the same ingredient and function as the metal-oxide surge arrester, protects the low-voltage electronic devices from lightning strike and switching surge by clamping the induced overvoltage to a level below the basic impulse insulation level of equipment. It has obvious advantages of high non-linearity, large energy absorption ability, and fast response. However, the MOV degrades gradually when subject to operating voltage, impulse current, and environment stress [1-3]. Left without check, the degradation progresses and finally results in the thermal runaway of the MOV, leading to the short-circuit and line-to-ground fault of electronic devices [4-5]. In addition, as its voltage level and rated peak surge current increase, the MOV has become increasingly important. Therefore, it is essential to monitor the condition of MOV to ensure itself and related equipment to operate safely and reliably.

The newly developed thermally protected metal-oxide varistor (TMOV), which is a new type of MOV, is composed of a thermally activated fuse connected in series with a conventional MOV. It can disconnect itself from the circuit in the event of overheating due to excessive impulse current or deterioration of MOV, preventing sustained follow current through the varistor. Until now, fewer studies have been carried out to investigate the deterioration characteristics of TMOV. In this paper, a harmonic analyzer for on-line condition monitoring of $\mathrm{MOV}$ was developed. Furthermore, the deterioration characteristics of the MOV and the newly developed TMOV under surge current and surge synchronized with operating voltage were investigated.

\section{Condition monitoring of MOV}

Various off-line and on-line methods, including the reference voltage [6-7], leakage current [8-14], power loss [15], voltage-current characteristic, thermal measurement [16], and electromagnetic field [17], have been presented for condition monitoring of MOV. The power loss and voltage-current characteristic methods include the detections of both leakage current and additional voltage signal, resulting in the complication of measuring circuit. The thermal measurement and electromagnetic field methods are suitable for on-line monitoring, however, complicated data analysis and expensive equipment are required.

The reference voltage is defined as the voltage across a MOV at the reference current that is selected by the manufactures [18]. It is at the keen-point of voltage-current curve of the MOV where the resistive leakage current becomes to be predominant in the total leakage current [16]. The reference current is usually in the range of $1 \mathrm{~mA}-10$ $\mathrm{mA}$ and is specified as $1 \mathrm{~mA}$ is this paper. As the varistor degrades, its reference voltage will increase or decrease depending on the polarity, and once the value changes by $10 \%$ of its initial value, the varistor is considered to be end of life and should be replaced [6-7]. However, the rated reference voltage varies depending on how the reference current is specified in the voltage-current curve provided by different manufactures [18]. In addition, the

* Department of Electrical and Electronics Engineering, Korea Maritime and Ocean University, Busan 49112, Republic of Korea, kilgs@kmou.ac.kr, ** HVDC Production Team, LSIS Co., Ltd, Busan, 46739, Republic of Korea, jhlee5@lsis.biz 


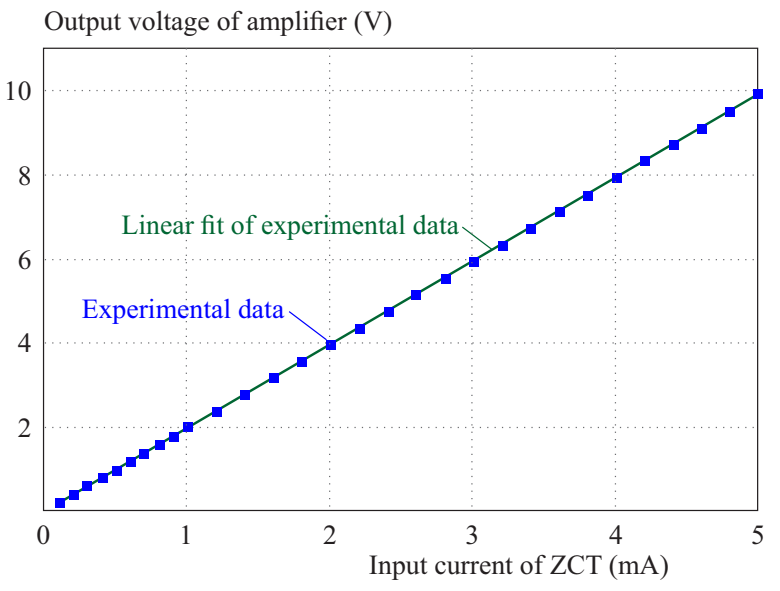

Fig. 1. Schematic diagram of the leakage current analyzer

MOV must be de-energized to carry out the measurement of reference voltage. Therefore, the reference voltage is a preferable indicator for assessment of MOV but is not suitable for on-line diagnosis of MOV.

Measurement and analysis of the leakage current have been regarded as the most effective method for condition monitoring of MOV. Due to the equivalent model of the series-parallel connected resistor and capacitor circuit, the total leakage current of a MOV is composed of a capacitive component which does not vary and a resistive component that increases with the degradation of MOV. Therefore, the resistive component represents the condition of a MOV. The amplitude of the capacitive leakage current is much higher than that of the resistive leakage current in the low conduction region [10-11]. Since the resistive current cannot be measured directly, off-line and on-line methods have been proposed for extracting the resistive component from the total leakage current. The circuit introduced in [11] can get the waveform of resistive leakage current by differentially amplifying the total leakage current and its capacitive component. However, considerable measuring error occurs when adjust the variable capacitor. The same result can be obtained by shifted current method, where the capacitive leakage current is generated by software $[10,14]$. This method is valid only when the resistive and capacitive leakage currents are orthogonal. However, the phase-shift is less than 90 owing to the microvaristor and microcapacitances within the bulk ZnO material [16]. Due to the nonlinearity characteristic of MOV, the total leakage current contains harmonic when sinusoidal voltage is applied. On-line condition monitoring of MOV can be achieved by analyzing harmonic contents of the total leakage current, which increase with the degradation as the resistive component but is easily to be acquired

\section{Development of a leakage current analyzer}

The rapid development of integrated circuit, signal acquisition and processing technique has contributed to the detection and analysis of leakage current. In this paper, a

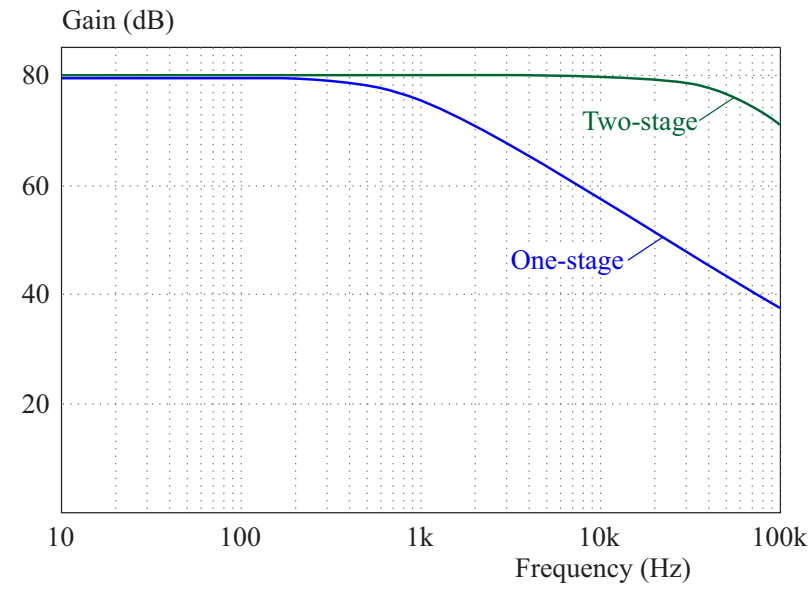

Fig. 2. Frequency responses of one-stage and two-stage amplifier

precise system consisting of an electrostatically shielded zero-phase current transformer (ZCT), a detection resistor $\left(R_{0}\right)$, an instrumentation amplifier (Amp), and a data acquisition unit (DAQ) was developed for on-line condition monitoring of MOV, which can analyze the total leakage current and its third harmonic content. Figure 1 shows the schematic diagram of the leakage current analyzer.

The leakage current of MOV is as small as a few hundreds of micro-ampere $(\mu \mathrm{A})$ at operating voltage. It should be amplified properly so that the DAQ can recognize it. The output voltage of amplifier depends on the resistance value of $\mathrm{R} 0$ and the gain of amplifier. The waveform of output voltage may be clamped if its amplitude is higher than the supply voltage of amplifier owing to over amplification. The optimal combination is $R_{0}$ of $500 \Omega$, amplifier gain of $80 \mathrm{~dB}$, and supply voltage of $15 \mathrm{~V}$.

The gain of amplifier decreases as the frequency of input signal increases. As the proposed analyzer is designated to analyze the third harmonic content whose frequency is $180 \mathrm{~Hz}$, a remarkable frequency response should be provided to avoid signal attenuation due to improper design. Figure 2 illustrates the frequency responses of a one-stage amplifier with a gain of $80 \mathrm{~dB}$ and a twostage amplifier, each of which has a gain of $40 \mathrm{~dB}$. The points where the gain starts to decrease are $130 \mathrm{~Hz}$ and $10 \mathrm{kHz}$, respectively. Therefore, the two-stage amplifier circuit was used.

Figure 3 shows the frequency response of the detection part, including the $\mathrm{ZCT}, R_{0}$, and amplifier. It can be seen that the detection part can measure the leakage current up to $700 \mathrm{~Hz}$ without attenuation, which covers the frequency of third harmonic content. The frequency at $-3 \mathrm{~dB}$ is $5500 \mathrm{~Hz}$. A calibration experiment was carried out to investigate the linear relationship between the input current of ZCT $\left(I_{\mathrm{L}}\right)$ and the output voltage of amplifier $\left(V_{\mathrm{O}}\right)$. The result is demonstrated in Fig. 4. The minute total leakage current can be detected with an adequate sensitivity and can be calculated by the following equation

$$
I_{\mathrm{L}}=\frac{V_{\mathrm{O}}}{1.98262} \quad(\mathrm{~mA})
$$




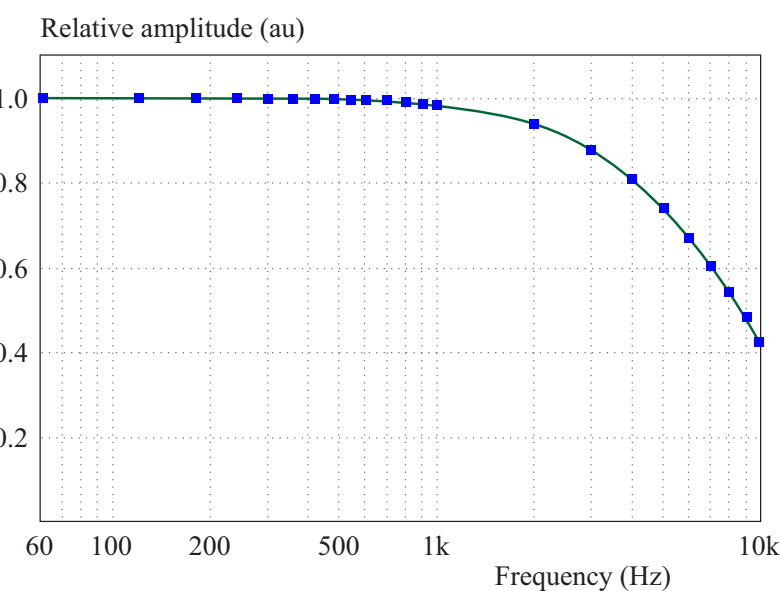

Fig. 3. Frequency response of detection part

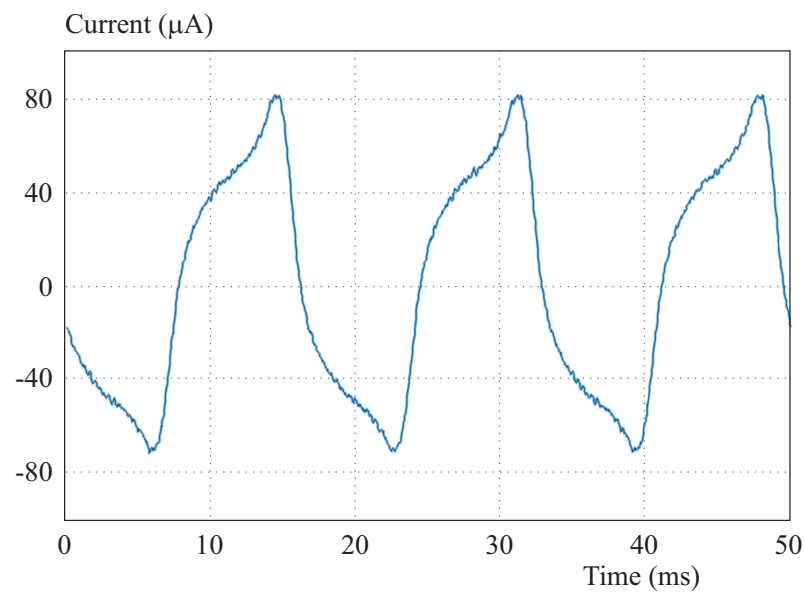

Fig. 5. Front panel of analysis algorithm

The conventional method uses the band-pass filter for extracting the harmonic content from total leakage current. The inherent frequency response characteristic and the bandwidth of filter make it difficult for exactly accurate measurement. In addition, several filters are essential for each order of harmonic content. Therefore, the software-based fast Fourier transform (FFT) was used. Only the coefficients corresponding to third harmonic

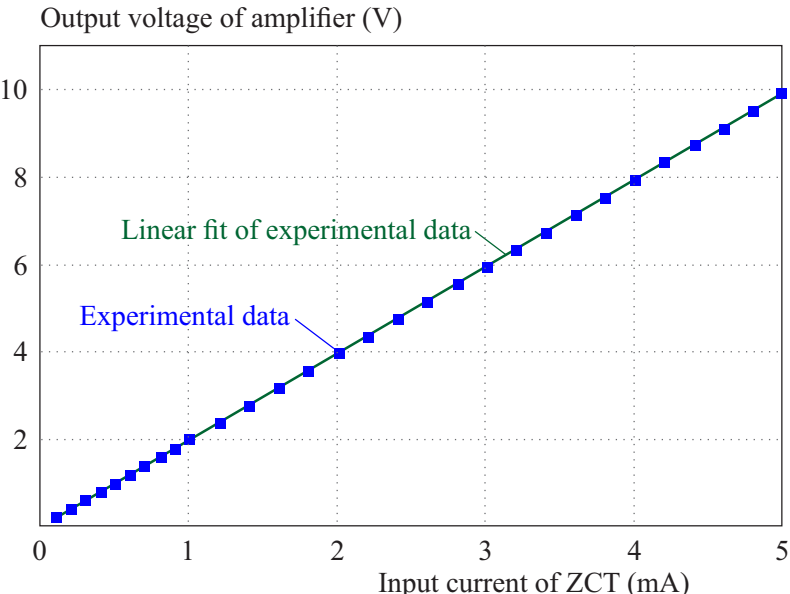

Fig. 4. Linear relationship between input current of ZCT and output voltage of amplifier

content were extracted and further used for signal reconstruction by inverse FFT. A software-based digital lowpass filter with a cut-off frequency of $5000 \mathrm{~Hz}$ was also used to eliminate the background noise. The analysis algorithm was developed based on LabVIEW program and its front panel is shown in Fig. 5. For automatically running the functions of standby, signal acquisition, analysis, and data storage, the standard state machine design pattern was built, which allows distinct states to operate in a programmatically determined dynamic sequence.

As the total leakage current and its third harmonic are voltage-and temperature-dependent, measuring uncertainties may occur when the system voltage and the environmental temperature change, leading to misjudge the condition of MOV. For instance, the leakage current of a seriously degraded MOV at lower voltage is smaller than that at the normal operating voltage; incorrect interoperation may be got if the influence of voltage variation is not taken into consideration. Therefore, multipliers were used to compensate the measured values to the standard operating condition, where the continuous operating voltage was $220 \mathrm{~V}$ and the ambient temperature was $20^{\circ} \mathrm{C}$. In order to investigate the change of leakage

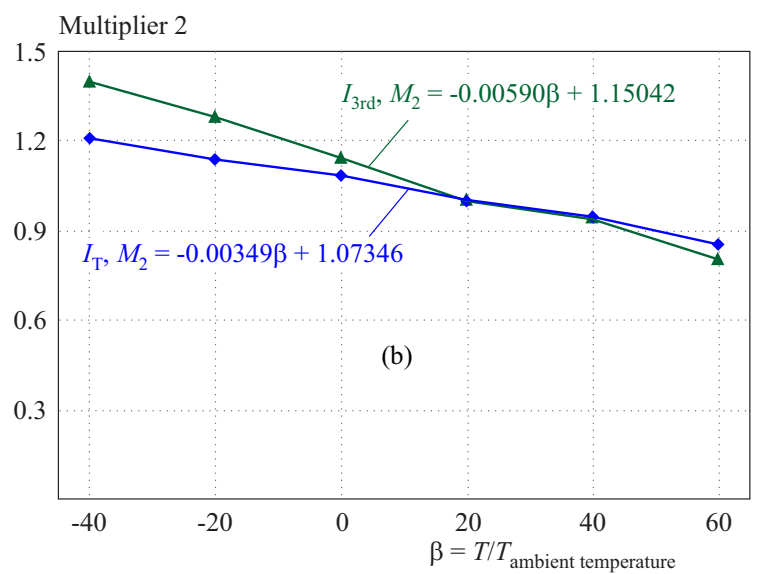

Fig. 6. Change of leakage current and third harmonic content: (a) - multiplier against operating voltage, (b) - multiplier against temperature 
Table 1. Specification of MOV and TMOV

\begin{tabular}{lc}
\hline Diameter & $14 \mathrm{~mm}$ \\
Rated peak current & $6 \mathrm{kA}$ \\
Maximum continuous operating voltage & AC: $275 \mathrm{~V}, \mathrm{DC}: 350 \mathrm{~V}$ \\
Reference voltage at $1 \mathrm{~mA}$ & $387 \mathrm{~V}-473 \mathrm{~V}$ \\
Clamping voltage at $8 / 20 \mu \mathrm{s}, 50 \mathrm{~A}$ impulse & $710 \mathrm{~V}$ \\
\hline
\end{tabular}

Table 2. Initial values of electrical characteristics of varistor samples

\begin{tabular}{lcccc}
\hline Varistor & Impulse & $\begin{array}{l}\text { MOV } \\
\text { Synchronized } \\
\text { impulse }\end{array}$ & Impulse & $\begin{array}{l}\text { TMOV } \\
\text { Synchronized } \\
\text { impulse }\end{array}$ \\
\hline$V_{\text {Ref }}(\mathrm{V})$ & 425 & 437 & 430 & 424 \\
$I_{\mathrm{T}}(\mathrm{A})$ & 41.58 & 42.37 & 42.14 & 43.7 \\
$I_{\mathrm{R}}(\mathrm{A})$ & 0.93 & 1.08 & 1.22 & 1.02 \\
$I_{3 \mathrm{rd}}(\mathrm{A})$ & 2.15 & 2.33 & 3.21 & 2.39 \\
\hline
\end{tabular}
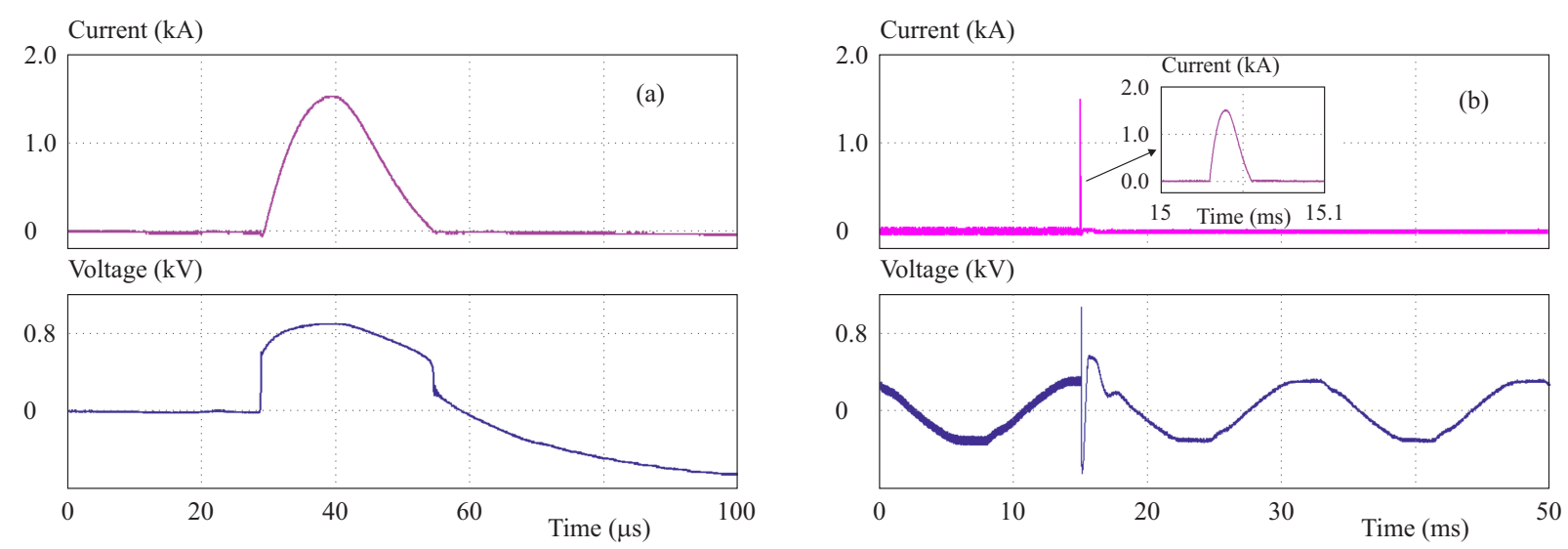

Fig. 7. Waveforms of current impulses for accelerated aging test: (a) $-8 / 20 \mu$ s lightning current impulse, (b) $-8 / 20 \mu$ s lightning current impulse synchronized with power-frequency voltage
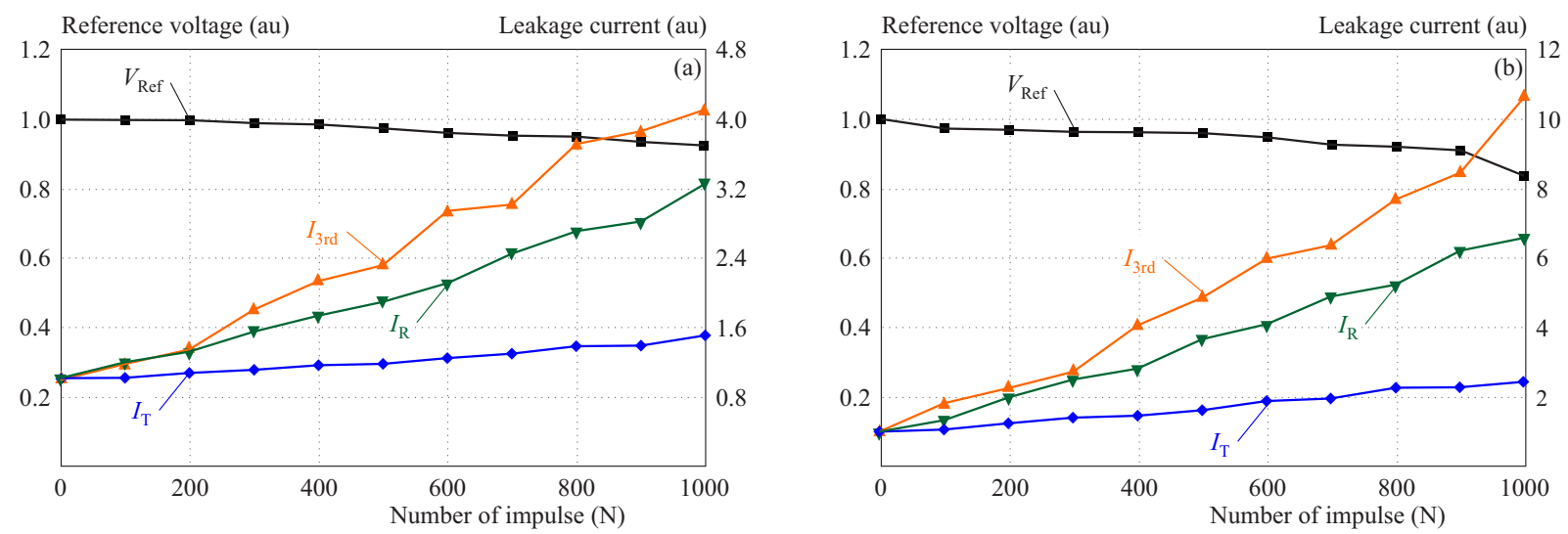

Fig. 8. Changes of electrical characteristics with impulse number of MOV: (a) - under lightning current impulse, (b) - under synchronized impulse

current with applied voltage and temperature, the AC 5 hours until its temperature reached the setting value; voltage was supplied by a power source that was free of harmonics; the MOV was placed in a thermo tank for and the total leakage current and third harmonic content were measured. The results are shown in Fig. 6 , by which 


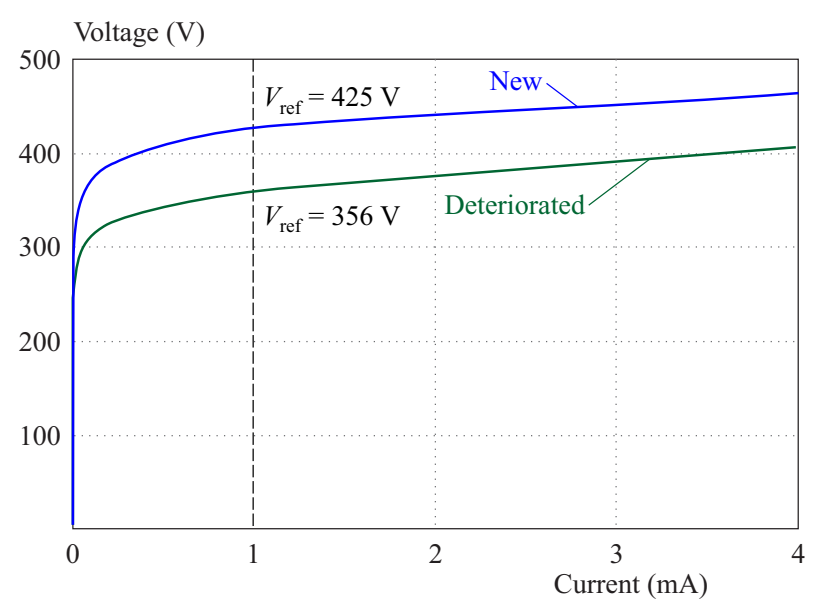

Fig. 9. Change of voltage-current characteristic with deterioration of MOV

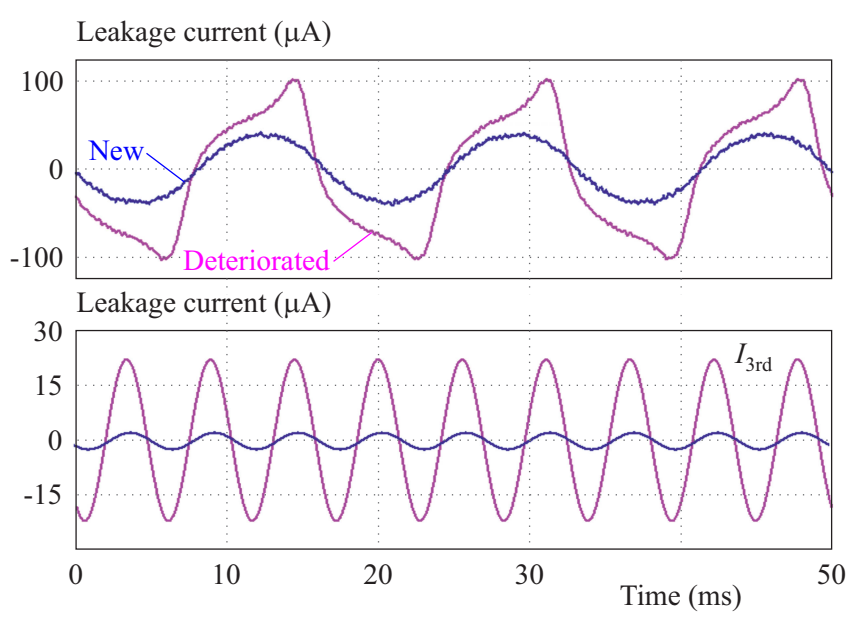

Fig. 10. Total leakage current and third harmonic component waveformes of MOV

the accuracy of proposed harmonic analysis system was further improved. The leakage current can be compensated by $I_{220 \mathrm{~V}, 20^{\circ} \mathrm{C}}=I_{\mathrm{m}} M_{1}(\alpha) M_{2}(\beta)$, where multiplier $1 M_{1}(\alpha)$ and multiplier $2 M_{2}(\beta)$ are the voltage and temperature factors, respectively. During on-line analysis, the voltage value can be easily obtained from the installed voltage meter or potential transformer and the temperature value can be acquired from a temperature sensor.

\section{Deterioration analyses of MOV and TMOV}

For investigating and comparing the deterioration characteristics of MOV and TMOV, samples with the same specification shown in Tab. 1 were used. The accelerated aging test was carried out using two types of current impulses: the $8 / 20 \mu$ s lightning current impulse and the $8 / 20 \mu$ s lightning current impulse synchronized with $220 \mathrm{~V}$ power-frequency voltage. Figure 7 shows the waveforms of current impulses and the voltage across varistors. The current impulse was applied to the varistors in groups of 100 times with an interval of 1 min every time.
After every group, the varistor was cooled for 2 hours at ambient temperature and the electrical characteristics in terms of reference voltage $\left(V_{\text {Ref }}\right)$, total leakage current $\left(I_{\mathrm{T}}\right)$, resistive leakage current $\left(I_{\mathrm{R}}\right)$, and third harmonic component of total leakage current $\left(I_{3 \mathrm{rd}}\right)$ were analyzed.

Table 2 shows the initial values of electrical characteristics of the MOV and the TMOV used for the accelerated aging test. Their change rates with number of impulse were analyzed.

\subsection{MOV}

The changes of electrical characteristics with the impulse number of MOV are shown in Fig. 8. After 1000 lightning current impulses were applied, the $V_{\text {Ref }}$ decreased to $92.4 \%$ of its initial value. The $I_{\mathrm{T}}, I_{\mathrm{R}}$, and $I_{3 \mathrm{rd}}$ increased to $1.51,3.27$, and 4.11 times of their initial values, respectively. When subjected to the lightning current impulse synchronized with the power-frequency voltage, the deterioration of MOV processed much faster. Its $V_{\text {Ref }}$ decreased to $83.8 \%$ of the initial value, and the $I_{\mathrm{T}}, I_{\mathrm{R}}$, as well as the $I_{3 \mathrm{rd}}$ increased to $2.45,6.59$, and 10.65 times of their initial values, respectively.

The voltage-current characteristics of a new and a deteriorated MOV are shown in Fig. 9, from which the decrease in reference voltage can be also seen. Although the MOV was still functional as its reference voltage decreased over $10 \%$, it should be replaced since thermal runway may occur owing to further deterioration.

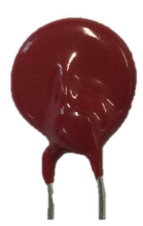

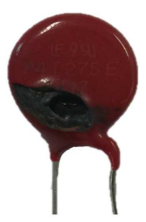

(b)

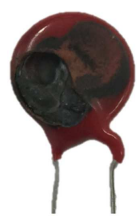

(c)
Fig. 11. Photographs of TMOV: (a) - new, (b) - failed under lightning current impulse, (c) - failed under impulse synchronized with power-frequency voltage

Deterioration of MOV resulted in the increase in its leakage current at the operating voltage, especially in the third harmonic component of total leakage current. Figure 10 shows waveforms of the total leakage current and the third harmonic component of a new and a deteriorated MOV. The total leakage current increased from $41.58 \mu \mathrm{A}$ to $101.75 \mu \mathrm{A}$ while the third harmonic component increased from $2.15 \mu \mathrm{A}$ to $22.88 \mu \mathrm{A}$. In addition, the waveform of total leakage current was seriously distorted due to the increase in resistive leakage current.

\section{$4.2 \mathrm{TMOV}$}

As the TMOV were subjected to the lightning current impulse and the impulse synchronized with powerfrequency voltage, its thermal fuse exploded at the 427 and 194 time of application, respectively. Figure 11 shows the photographs of new and failed TMOVs. Since more 


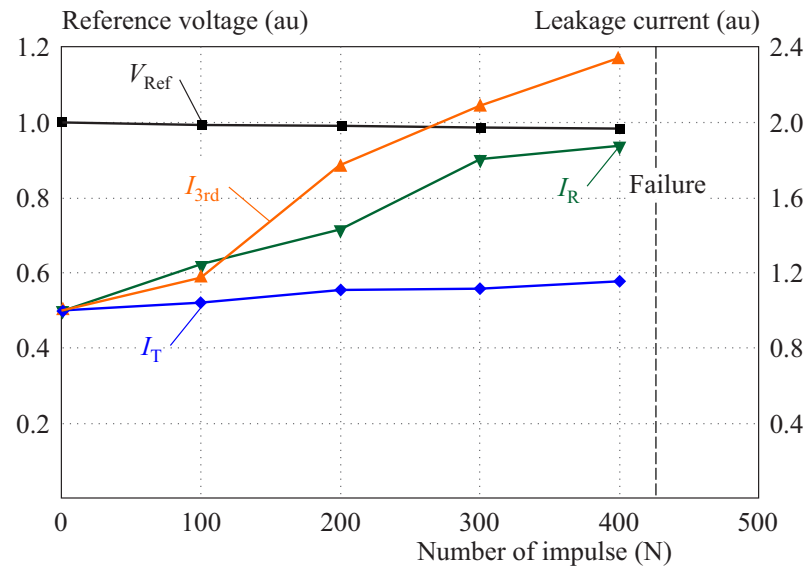

Fig. 12. Changes of electrical characteristics with impulse number of TMOV under lightning current impulse

energy was absorbed under the synchronized impulse, the TMOV exploded seriously.

Figure 12 shows the changes of electrical characteristics with the impulse number of TMOV under lightning current impulse. Compared with that of the MOV, the electrical characteristics of TMOV did not change greatly before the explosionof thermal fuse, resulting in the difficulty in condition monitoring. The phenomenon was much worse for the TMOV under impulse synchronized with power-frequency voltage. It is recommended by the manufactures that the TMOV can substitute the conventional MOV and provide enhanced reliability by disconnecting the damaged varistor from the circuit. In addition, a subsidiary circuit including an LED or an optical coupler can indicate that the TMOV has failed and should be replaced. However, based on the experimental result, it is difficult to real-timely diagnose the condition of energized TMOV in advance of its failure by monitoring the third harmonic content of total leakage current.

\section{Conclusions}

In this paper, for the purpose of monitoring the condition of MOV in advance of its failure, an analyzer was developed to detect and analyze the total leakage current and the third harmonic component of total leakage current. The analyzer was composed of a ZCT, a detection resistor of $500 \Omega$, a two-stage amplifier with a gain of $80 \mathrm{~dB}$ as well as an operating frequency up to 10 $\mathrm{kHz}$, and a DAQ. The third harmonic component was extracted using the FFT method instead of the conventional band-pass filter. The multipliers were used to compensate the voltage- and temperature-dependent measuring uncertainties, resulting in an improvement in the accuracy of the analyzer.

The accelerated aging test was carried out by applying the $8 / 20 \mu$ s lightning current impulse and the lightning current impulse synchronized with $220 \mathrm{~V}$ power-frequency voltage to investigate and compare the deterioration characteristics of the MOV and the newly developed TMOV. From the results, deterioration of varistor resulted in the decrease in reference voltage and the increase in total leakage current, resistive leakage current, and third harmonic component. The deterioration of MOV processed faster under the lightning current impulse synchronized with power-frequency voltage. In addition, the TMOV was much more vulnerable when subjected to impulse as its thermal fuse exploded. Although the TMOV can disconnect itself from the circuit in the event of overheating, it has a shorter lifetime compared with MOV. It is also difficult to diagnose the TMOV before its failure since the electrical characteristics do not change greatly.

The results from this paper are expected to improve the reliability of varistors used in the low-voltage electronic circuits and to provide suggestions to users for selecting the optimal varistors.

\section{REFERENCES}

[1] G. H. Chen, J. L. Li, X. Chen, X. L. Kang and C. L. Yuan, "Sintering temperature dependence of varistor properties and impedance spectroscopy behavior in $\mathrm{ZnO}$ based varistor ceramics", J. Mater. Sci. Mater. Electron., 26, 2389-2396 (2015).

[2] C. A. Christodoulou, M. V. Avgerinos, L. Ekonomou, I. F. Gonos and I. A. Stathopulos, "Measurement of the resistive leakage current in surge arresters under artificial rain test and impulse voltage subjection", IET Sci. Meas. Technol., 3, 256-262 (2009).

[3] C. Zhang, D. Zhou, W. Lu and Y. Hu, "Microstructure and properties of low-voltage $\mathrm{ZnO}$ varistor ceramics", J. Mater. Sci. Mater. Electron., 12, 357-360 (2001).

[4] IEC 61643-331, Components for low-voltage surge protective devices - Part 331: Specification for metal oxide varistors (MOV), 1st edn.(IEC, Geneva, 2003), pp.17-25.

[5] G. M. Wang, S. J. Kim, S. J. Park, G. S. Kil and H. K. Ji, "Optimal design of a follow current disconnector for DC arresters in traction vehicles", Trans. Electr. Electron. Mater., 17, 289-292 (2016).

[6] R. B. Standler, Protection of electronic circuits from overvoltages, 1st end. (John Wiley \& Sons, New York, 1989), pp.133-145.

[7] E Limited HEMP Filter Maintenance and Monitoring, https:// www.mpe.co.uk, Accessed 4 December 2012.

[8] J. Lundquist, L. Stenstrom, A. Schei and B. Hansen, "New method for measurement of the resistive leakage currents of metal-oxide surge arresters in service", IEEE Trans. Power Del., 5, 1811-1822, (1990).

[9] A. Haddad, J. Fuentes-Rosado, D. M. German and R. T. Waters, "Characterisation of $\mathrm{ZnO}$ surge arrester elements with direct and power frequency voltage", IEE Proc. A. Phys. Sci. Meas. Instrum. Manage. Educ., 137, 269-279 (1990).

[10] B. H. Lee and S. M. Kang, "A new on line leakage current monitoring system of Zno surge arresters", Mater. Sci. Eng., B.119, 13-18 (2005)

11] S. Shirakawa, F. Endo, H. Kitajima, S. Kobayashi, K. Kurita, K. Goto and M. Sakai, "Maintenance of surge arrester by a portable arrester leakage current detector", IEEE Trans. Power Del., 3, 998-1003 (1998).

[12] P. Papliñski and J. Wañkowicz, "Application of leakage current parameter for technical diagnosis of surge arresters", IEEE Trans. Dielect. Electr. Insul., 23, 3458-3465 (2016).

[13] M. Khodsuz and M. Mirzaie, "Harmonics ratios of resistive leakage current as metal oxide surge arresters diagnostic tools", Meas., 70, 148-155 (2015). 
[14] Z. Abdul-Malek, Novizon and Aulia, "A new method to extract the resistive component of the metal oxide surge arrester leakage current", IEEE International Conference on Power and Energy, 399-402 (2008).

[15] L. T. Coffeen and J. E. McBride, "High voltage ac resistive current measurements using a computer based digital watts technique", IEEE Trans. Power Del., 6, 550-556 (1991).

[16] C. Heinrich and V. Hinrichsen, "Diagnostics and monitoring of metal-oxide surge arresters in high-voltage networks-comparison of existing and newly developed procedures", IEEE Trans. Power Del., 16, 138-143 (2001).

[17] K. L. Wong, "Electromagnetic emission based monitoring technique for polymer $\mathrm{ZnO}$ surge arresters", IEEE Trans. Dielect. Electr. Insul., 13, 181-190 (2006).

[18] IEC 60099-4 and Surge arresters - Part 4:, "M", al-oxide surge arresters without gaps for a.c. systems, 3rd edn.(IEC, Geneva, 2014), pp.29-55.

Received 29 July 2017

Guoming Wang was born in Liaoning, China in 1990. He received the BEng degree from Division of Engineering, Dali University, China in 2013 and the MEng. degree from Division of Electrical and Electronics Engineering, Korea Maritime and Ocean University, Korea in 2015. He is currently a PhD candidate at Division of Electrical and Electronics Engineering, Korea Maritime and Ocean University. His research interests are in the area of high voltage engineering, diagnosis of power facilities, and arc detection.
Woo-Hyun Kim was born in Ulsan, Korea in 1992. He received the BEng degree from Division of Electrical and Electronics Engineering, Korea Maritime and Ocean University, Korea in 2017. He is currently a MEng candidate at Division of Electrical and Electronics Engineering, Korea Maritime and Ocean University. His research interests are in the area of high voltage engineering and diagnosis of power facilities.

Jong-Hyuk Lee was born in Busan, Korea in 1973. He received the BEng and MEng degree from Division of Electrical and Electronics Engineering, Korea Maritime and Ocean University, Korea in 1999 and 2001, respectively. He is currently a $\mathrm{PhD}$ candidate at Division of Electrical and Electronics Engineering, Korea Maritime and Ocean University and is a team leader at HVDC Production Team, LSIS Co., Ltd. His research interests are in the area of diagnosis of power facilities and manufacture of HVDC apparatus.

Gyung-Suk Kil (Professor) was born in Incheon, Korea in 1962. He received the BEng, MEng, and PhD degrees from Inha University, Korea in 1984, 1987, and 1996, respectively. Since then, he has been a professor at the Division of Electrical and Electronics Engineering, Korea Maritime and Ocean University. He worked as a visiting professor at Cardiff University in 2003. His research interests are in the area of high voltage engineering, diagnosis of power facilities, grounding technique, lightning protection, and arc detection. He is a member of the IEEE Dielectrics and Electrical Insulation Society. 